CASE STUDY

\title{
Breaking the Iron Triangle at The University of Central Florida
}

August 26, 2015

J essie Brown

Martin Kurzweil

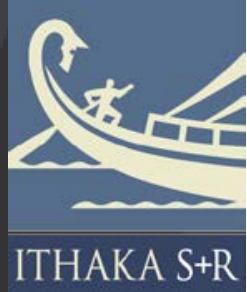




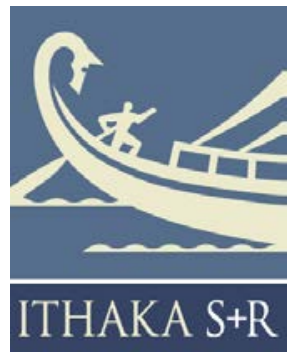

Ithaka $\mathrm{S}+\mathrm{R}$ is a strategic consulting and research service provided by ITHAKA, a not-for-profit organization dedicated to helping the academic community use digital technologies to preserve the scholarly record and to advance research and teaching in sustainable ways. Ithaka $\mathrm{S}+\mathrm{R}$ focuses on the transformation of scholarship and teaching in an online environment, with the goal of identifying the critical issues facing our community and acting as a catalyst for change. J STOR, a research and learning platform, and Portico, a digital preservation service, are also part of ITHAKA.

Copyright 2015 ITHAKA. This work is licensed under a Creative Commons Attribution-NonCommercial 4.0 International License. To view a copy of the license, please see http://creativecommons.org/licenses/by-nc/4.0/.

ITHAKA is interested in disseminating this case study as widely as possible. Please contact us with any questions about using the case study: research@ithaka.org. 


\section{Introduction}

Scanning the social needs and economic realities faced by institutions of higher education in 2008, J ohn Immerwahr described an "iron triangle" constraining colleges and universities. Immerwahr suggested that the three points of this triangle-cost, quality, and access - exist in an "unbreakable reciprocal relationship, such that any change in one will inevitably impact the others." According to this logic, making a college or university more accessible or trying to increase the quality of instruction would necessarily drive up institutional costs. Conversely, reducing expenditures would inevitably make an institution less accessible and undermine the quality of the education that a student could receive there. ${ }^{1}$

\section{For twenty years, the University of Central Florida... has sought to break this iron triangle by reducing cost, improving quality, and enhancing access simultaneously.}

For twenty years, the University of Central Florida (UCF), a public university of nearly 61,000 students in Orlando, has sought to break this iron triangle by reducing cost, improving quality, and enhancing access simultaneously. With a 144 percent increase in undergraduate enrollment, a graduation rate approaching 70 percent, close institutional attention to teaching and learning, and tuition and fees of just over $\$ 6,000$ per year, it has arguably succeeded.

To improve and scale instruction, UCF has pursued two main strategies. First, it has strategically developed its own resources-through satellite campuses, online instruction, and rigorous faculty development-to extend its internal instructional capacity. Second, it has partnered with other institutions in the region to develop and integrate its external instructional capacity.

From its early days, UCF sought to extend its internal capacity through a network of Regional Campuses-remote sites offering UCF bachelor's and master's degrees, co-

${ }^{1}$ John Immerwahr, Jean Johnson, Paul Gasbarra, "The Iron Triangle: College Presidents Talk About Costs, Access and Quality," The National Center for Public Policy and Higher Education and Public Agenda (October 2008), http://www.highereducation.org/reports/iron triangle/IronTriangle.pdf. 
located on the campuses of state colleges that offer primarily associate's degrees. For the past twenty years, UCF has supplemented this physical extension with a vast, virtual extension of its instructional reach through technology. Now, nearly 78 percent of all UCF students take online or hybrid courses and 38 percent of all credits are earned online. To ensure the quality of online and blended instruction, Online@UCF is grounded in a robust training and support program in instructional design. ${ }^{2}$

The second strategy has focused on supporting and taking better advantage of instructional capacity at other institutions, thereby smoothing the pathway between associate's degree and bachelor's degree while ensuring that a quality education remains accessible to increasing numbers of central Florida students. Through DirectConnect to UCF, UCF guarantees admission to all associate's degree graduates of Daytona State College, Eastern Florida State College, Lake-Sumter College, Valencia College, and Seminole State College. DirectConnect to UCF goes far beyond the admission guarantee, however: administrators, staff, and faculty at UCF and its partners have worked closely to improve and coordinate instruction and advising, as well. More than half of UCF students transferred there after earning an associate's degree elsewhere, and the majority of those transfers are from DirectConnect to UCF partners. The graduation rate of transfer students is virtually the same as that of students who start at UCF.

To learn more about UCF's transformation, we spent two days in April 2015 meeting with thirteen staff members and administrators on UCF's main campus. ${ }^{3}$ In addition, we attended a portion of UCF's College Access Summit, an annual event in which advisors, support staff, administrators and faculty from UCF and its partner colleges engage in structured discussions to coordinate curriculum, advising, and logistics to enhance DirectConnect to UCF. ${ }^{4}$ We also reviewed documentation on UCF's initiatives, such as research on Online@UCF published by UCF faculty and administrators, including several of our interviewees.

UCF's efforts to break the iron triangle are notable for their emphasis on instructional quality and faculty development. This approach requires patience and long-term thinking. It takes years to engage a critical mass of participating faculty, to build their capacity, and to see their efforts affect large numbers of students and bring more faculty

\footnotetext{
${ }^{2}$ Headcount Statistics, Academic Year 2014-2015, University of Central Florida Center for Distributed Learning (provided by Joel Hartman).

${ }^{3}$ We are deeply grateful to Joel Hartman, Jeff Jones, and Tom Cavanagh for coordinating our visit and sharing their insights regarding UCF's initiatives. The full list of interviewees is in the Appendix.

${ }^{4}$ To see a summary of this year's summit, see "Summary College Access Summit 2015," College Access Initiative, http://www.collegeaccess.ucf.edu/download/attachments/360479/College\%20Access\%202015\%20Mins.docx?version=1\& modificationDate $=1431957448383 \& a p i=v 2$.
} 
into the fold. But UCF was not attempting merely to change policy, organizational responsibility, or funding, each of which can happen at the stroke of a pen. At the root of this transformation has been an attempt to change culture among its own faculty and staff and among those at its partner institutions. The gestation for this sort of change has been longer, but the results are substantial and deeply engrained.

\section{At the root of this transformation has been an attempt to change culture among its own faculty and staff and among those at its partner institutions.}

\section{Origins and Operations}

Administrators at UCF enjoy telling the story of how the institution began as little more than a 1,227-acre sandpit with a dirt road, founded in 1963 to support Florida's growing space program. With fewer than 2,000 students, the institution, then known as Florida Technological University, was built on a plot 12 miles northeast of downtown Orlando. This location was chosen because it was the most accessible to the largest number of people in the east central Florida area. ${ }^{5}$

Since these humble beginnings, UCF has grown immensely: in the fall of 2014, UCF enrolled 60,821 students in 210 different degree programs. ${ }^{6}$ Throughout its history, UCF has remained focused on serving the workforce needs of the state and providing access to higher education to residents in central Florida. As Florida's workforce needs changed, the institution began to offer a broader array of liberal arts, business, and education programs, and in 1978 the university's second president, Trevor Colbourn, changed the institution's name to University of Central Florida to fit its changing mission. Additionally, as the region's population expanded and state plans for higher education called for increased access, the institution grew its operations, enrollment, and offerings, and began to search for innovative ways to serve more students in the central Florida region.

\footnotetext{
${ }^{5}$ See "History," UCF50, http://www.ucf.edu/50/history/.

6 "UCF Current Facts," UCF Institute of Knowledge Management (July 24, 2015), https://ikm.ucf.edu/files/2015/07/UCFCurrent-Facts-07.24.15.pdf.
} 


\section{Regional Campuses}

One of the earliest initiatives UCF implemented to serve more students within the region was its Regional Campuses. Housed in joint-use facilities on the campuses of two-year state colleges, UCF's regional campuses are satellite locations where students can earn a UCF degree without traveling far from home. Their mission was and continues to be "to provide access to quality higher education for students who, because of time, distance, preference, or life circumstance, might not otherwise have the opportunity to pursue a baccalaureate or graduate degree." ${ }^{\prime 7} \mathrm{UCF}$ now has nine regional campuses across central Florida, which offer, in total, 22 Bachelor's degree programs, 15 minors, and 5 Graduate programs. ${ }^{8}$ UCF's Regional Campuses have allowed UCF to extend its degree granting programs beyond the physical boundaries of its main campus, eroding some of the geographical and scheduling barriers that might otherwise prevent students from enrolling or succeeding.

\section{UCF's Regional Campuses have allowed UCF to extend its degree granting programs beyond the physical boundaries of its main campus, eroding some of the geographical and scheduling barriers that might otherwise prevent students from enrolling or succeeding.}

\section{Online@UCF}

Like regional campuses, Online@UCF has allowed the university to increase its internal instructional capacity and serve more students without being constrained by the physical limits of its main campus. Launched in 1995, Online@UCF was prompted by a set of concerns related to access, quality, and scale. First, leading up to its ten-year Southern

\footnotetext{
${ }^{7}$ A variety of Florida research universities have joint-use facilities on state college campuses where they offer both instruction and support services. See, for example, Florida International University's I-75 in Miramar (http://broward.fiu.edu/about/index.html) and University of South Florida in Lakeland (http://usfweb2.usf.edu/lakeland/).

8 In 1982, UCF opened its first regional campus at Eastern Florida State College (then Brevard Community College), and in 1987 it constructed a joint-use facility at Daytona State College (then called Daytona Beach Community College). In the 1990s, UCF constructed joint-use facilities at Eastern Florida State College in Palm Bay, Lake Sumter State College, Seminole State College and Valencia College's various locations. For more on Regional Campuses, see the Regional Campus website and strategic plan at http://regionalcampuses.ucf.edul.
} 
Association of Colleges and Schools Commission on Colleges (SACSCOC) accreditation review, UCF conducted a major self-study that revealed a need for clarification and improvement with respect to a number of academic policies and programs, including distance learning. Second, UCF was in the early stages of a plan to increase enrollment substantially, and online solutions presented an opportunity to do so in a cost-effective way. Finally, some of the community colleges from which students UCF students transferred were experimenting with e-learning tools and techniques, and those students' praise for and comfort with the modality encouraged UCF to take it up as well.

\section{[A]n online learning model emerged that was based on intensive faculty development, an emphasis on academic quality, well-designed courses across all departments and modalities, and deep formative and summative} assessment.

To determine the course that UCF would take with online learning, President J ohn Hitt and Vice Provost J oel Hartman convened a faculty committee to lay the groundwork for Online@UCF. Informed by research conducted by a professor and graduate student in UCF's College of Education, an online learning model emerged that was based on intensive faculty development, an emphasis on academic quality, well-designed courses across all departments and modalities, and deep formative and summative assessment. From these principles emerged two initiatives: UCF's Center for Distributed Learning (CDL), which manages training and supports course design and development, and, a couple of years later, the Research Initiative for Teaching Effectiveness (RITE), which evaluates the online course initiative and provides support for faculty research related to online teaching and learning. ${ }^{9}$

In the fall of 1996, UCF launched a small set of online courses in a variety of disciplines. Though these courses were originally targeted toward nonresident students, early assessments revealed that most of the students in the courses resided on the main

\footnotetext{
${ }^{9}$ For more information, see the Center for Distributed Learning's website, https://online.ucf.edu/about/ and the Research Initiative for Teaching Effectiveness's website, https://online.ucf.edu/research. Though research and assessment were a crucial part of Online@UCF from its inception, RITE did not become a formal department until 1999. See Patsy Moskal, "Dancing with a Bear: One University's Experience with Evaluating Blended Learning," Journal of Asynchronous Learning Networks 13:1(2009), 65-74.
} 
campus. To accommodate these findings, CDL began training faculty to design blended modality courses, which mix classroom and online course delivery, and launched the first set of such courses in 1997. In addition to fully online and blended courses, Online@UCF now includes video lecture capture courses, which are particularly popular with the colleges of business and engineering. Currently, Online@UCF includes 18 baccalaureate degree programs, 27 graduate degree programs, 34 online graduate certificate programs, and 28 undergraduate minors. As of Summer 2015, 1,774 different fully online courses and 1,628 different blended courses had been offered at UCF. ${ }^{10}$

While Online@UCF has allowed UCF to scale enrollment growth substantially, administrators at UCF emphasize that, from the beginning, Online@UCF has been an academic endeavor focused on expanding and improving UCF's instructional capacity. As such, a substantial portion of CDL's operations are dedicated to helping faculty design and develop their courses. CDL's core offering is IDL 6543, an eight-week, eighty-hour, blended-format faculty development program that UCF has been offering and iteratively revising over the past twenty years. The course is required for faculty from all disciplines who plan to create and teach original online or blended courses. (CDL offers morefocused, less-intensive trainings for faculty who are taking over an existing online course.)

\section{One of the key tenets of [UCF's Center for Distributed Learning] is that an online course should not simply be a "conversion" of an existing course, but should instead involve taking apart and "rebuilding" the original course to achieve its learning goals with new teaching tools.}

In addition to providing technical training, IDL 6543 engages faculty in thinking about the teaching and learning objectives for their courses. One of the key tenets of CDL is that an online course should not simply be a "conversion" of an existing course, but should instead involve taking apart and "rebuilding" the original course to achieve its learning goals with new teaching tools. In order to achieve these ends, each faculty member enrolled in IDL 6543 is paired with a trained instructional designer who serves as a coach during the training and continues to support the faculty member afterwards.

10 "Summary Data," University of Central Florida Center for Distributed Learning, Summer 2015 analysis, provided by Joel Hartman. 
These instructional designers, most of whom have teaching experience and advanced degrees in instructional design, help faculty members think through all aspects of course design, including but not limited to which technological tools can best serve course objectives.

CDL also works with deans to determine how online learning can help departments and colleges expand and improve their instructional reach. For example, because the College of Nursing serves a large number of working students, CDL has helped it develop flexible, fully online degree programs. Similarly, CDL has helped the College of Arts and Humanities create online versions of popular general education courses to alleviate bottlenecks for residential students. CDL's academic planning team has also analyzed data on online enrollments to help departments identify degree programs for which a fully online option could be offered with only a few more online courses.

The Research Initiative for Teaching Effectiveness, which is a unit within CDL, oversees the evaluation of online programs and provides support for faculty research on online teaching. Though implemented as a formal program in 1999, RITE's director, Chuck Dziuban, was recruited to provide summative and formative assessment and evaluation services for Online@UCF when the program launched in 1995. In addition to evaluating student success and satisfaction for courses of all modalities, RITE provides support to faculty conducting projects on the impact of technology in their classes. At any given time, RITE supports thirty to forty faculty members on their own research projects. Many departments give publication and presentation credit for faculty research on teaching and learning, reinforcing this culture of inquiry.

In light of its longstanding and focused work on online instructional design, UCF has made efforts to serve as a resource on the subject to other institutions. As noted above, in 2010, along with the American Association of State Colleges and Universities, CDL received a Gates Foundation-funded Next Generation Learning Challenge grant from EDUCAUSE to develop the Blended Learning Toolkit, an open resource for educational institutions interested in developing or expanding their blended learning initiatives. For the past two years, CDL has built on these efforts and partnered with EDUCAUSE to offer a five week MOOC for faculty developing blended courses. ${ }^{11}$ Each time the course was offered, nearly 3,000 faculty members across the nation signed up, and more than 80 paid an additional fee each session to have their work reviewed by a UCF instructional designer.

${ }^{11}$ See UCF/EDUCAUSE Blendkit 2015," http://www.educause.edu/eli/events/eli-ucf-mooc. 
UCF continues to innovate in the online space to broaden its instructional capacity and increase its instructional quality. It is partnering with state colleges to build $2+2+2$ pathways for students that consist of two years at a local state college, two years earning a bachelor's degree through Online@UCF, and two years earning a graduate degree through Online@UCF. In addition, UCF has begun to experiment with badging for competencies, and is piloting an adaptive learning platform in nursing, psychology and math courses. Though it is still in a pilot phase, UCF administrators are hopeful that, if executed correctly, these courses' self-adaptive nature will improve student success rates and learning outcomes.

\section{DirectConnect to UCF}

If Regional Campuses and Online@UCF have allowed UCF to extend its internal capacity without compromising instructional quality, then DirectConnect to UCF has provided an avenue for UCF to leverage instructional capacity at other institutions in onder to ensure that students receive a high-quality, affordable education. In 1998, a master plan released by the Florida Postsecondary Education Planning Commission authorized select community colleges to become "state colleges" and offer bachelor's degrees. ${ }^{12}$ Because UCF was (and is) a major recipient of associate's degree transfers, this change had the potential to erode its student pipeline. Moreover, UCF's increasing focus on research and improving reputation meant that it was attracting more applicants and, even as its enrollment grew, becoming more selective. Leaders at UCF worried that these changes would dilute UCF's regional focus and make the institution less accessible to Florida students.

To address these issues, President J ohn Hitt of UCF, President Sanford Shugart of Valencia College, and Dave Harrison, then-Vice Provost of Regional Campuses at UCF, assembled the presidents of all central Florida state colleges. Leaders at four of these institutions-Eastern Florida State College (then Brevard Community College), Lake Sumter State College, Seminole State College, and Valencia College-developed a memorandum of agreement in 2005, "indicating their commitment to a regional strategy for higher education designed to expand across, meet the extraordinary demands for growth, and provide for a competitive, skilled workforce in central Florida." In addition to guaranteeing admission to UCF for associate degree holders from these four

\footnotetext{
${ }^{12}$ See "Challenges and Choices: The Master Plan for Florida Postsecondary Education," Report and Recommendations by the Florida Postsecondary Education Planning Commission (1998), http://files.eric.ed.gov/fulltext/ED423801.pdf. In addition to authorizing certain community colleges to become "state colleges," the master plan called for state universities to enhance their research activities and increase enrollment; an increase in the number of joint-use facilities; and an increase in the use of distance learning and instructional technology.
} 
consortium colleges, the memorandum committed to a variety of measures to ensure that transfer students would receive a high quality education and enjoy a smooth process of transition. In 2014, the partner institutions re-signed this memorandum and introduced Daytona State College into the consortium. ${ }^{13}$

Though this memorandum formalized DirectConnect to UCF and institutionalized guaranteed admissions for students from participating schools, it did not create a new system from scratch. Florida already had a strong $2+2$ program that guaranteed graduates of state colleges admissions to one of Florida's 11 public universities (though not specifically UCF). Additionally, UCF already enrolled a large number of transfer students from regional schools. The 2005 agreement solidified some of these arrangements, institutionalizing a more regionally focused $2+2$ program and creating incentives for more structured pathways and more robust support programs. Because DirectConnect to UCF was introduced in an institutional (and state) context that already embraced access and partnerships, there was little friction in its implementation.

\section{What makes DirectConnect to UCF different from other $2+2$} policies ... is the constellation of initiatives and efforts that ensure transfer students are well prepared for UCF and graduate at the same rates as first time in college (FTIC) students.

What makes DirectConnect to UCF different from other $2+2$ policies (including the Florida policy that preceded it) is the constellation of initiatives and efforts that ensure transfer students are well prepared for UCF and graduate at the same rates as first time in college (FTIC) students. For example, UCF uses its joint-use facilities at partner colleges to provide admission, enrollment, financial assistance, and advising support to students contemplating transfer. It has also worked on curriculum alignment initiatives with its state college partners to ensure that transfer students are well-prepared for UCF departmental courses in their chosen major and do not need to retake courses. Because many transfer students live farther from UCF's main campus than FTIC students do and often have more competing life demands, UCF's Regional Campuses and Online@UCF

${ }^{13}$ For more on DirectConnect to UCF and its development, see Saundra Amrhein, "Where Dreams Come True," Politico Magazine (June 18, 2015), http://www.politico.com/magazine/story/2015/06/orlando-what-works119159.html\#.VZK4XVeXFwU. 
make it easier for transfer students to successfully complete courses once they have transferred.

Perhaps the most notable feature of DirectConnect to UCF-and the key to ensuring that the program delivers quality along with access -is the culture of collaboration that supports it. For instance, each year, administrators and support staff from UCF and its partner colleges convene at a College Access Summit, where they break into disciplinespecific working groups to discuss how departments at UCF and partner colleges can work together to ease the transition for students who plan to major in their department's fields. We had the opportunity to observe part of the 2015 summit, and were impressed by the collaborative way in which individuals across institutions tackled questions and problems (some of which are discussed on our section on "Remaining Challenges"). In addition to the College Access Summit, UCF and its partner colleges participate in a shared data resource group, develop curricula together, and collaborate on grants. Crossinstitutional working groups for enrollment services, advising, student affairs, academic affairs, financial aid, and registrars meet multiple times each year.

DirectConnect to UCF has been heralded as one of the largest and most successful transfer pathways in the nation, and various program staff have received accolades for their work. One recent, award-winning initiative is the Pathways program. Pathways is the product of a recent Florida policy change that required all students declare a major by 30 hours to avoid excess credit hours. Prior to this policy, DirectConnect to UCF advisors would have little interaction with students before they reached 30 credit hours. Now, utilizing frameworks grounded in student development competencies, academic and career planning, and academic and social integration, students receive DirectConnect to UCF advising services through the Pathways program as soon as they enter a partner college.

While Pathways is best described as an advising model, technology plays a large role in its administration. Students engage with a guided instructional program in Canvas, UCF's learning management system, in which they complete modules helping them think through degree choice and financial and career planning. "Assessments" in the program ask students to reflect upon what they learn in the modules, and Pathway teams (which consist of both UCF and partner school advisors) combine students' answers with other relevant data to assess which paths might best fit a student.

\section{Evidence of Impact}

In the past twenty years, UCF has experienced dramatic increases in enrollment. Between 1995 and 2014, UCF nearly doubled its undergraduate enrollment from 21,519 
to 52,539. The student population now has a larger share of Black and Hispanic students (between 2001 and 2013, the share of students who are Black or Hispanic increased from 19 percent to 33 percent. UCF also now serves a larger share of low-income students: between 2009 and 2013, the share of students in the entering class eligible for Pell grants increased from 24 percent to 31 percent.

At the same time that it grew larger and more diverse, UCF significantly improved its completion rate. Between 2001 and 2014, UCF's six-year graduation rate for first-time, full-time students from 57 percent to 68 percent. The five-year graduation rate of transfer students was 67 percent for the cohort that enrolled in 2009. This is nearly identical to the 68 percent six-year graduation rate for the FTIC cohort of 2008.

As it has expanded and improved completion rates, UCF has maintained relatively low tuition and expenditures per student, even in the face of reduced state funding. From 2006 to 2013, state appropriations per full-time student decreased from $\$ 6,300$ to $\$ 3,800$, and educational and related expenditures increased from only $\$ 8,438$ per FTE in 2007 to $\$ 9,137$ per FTE in 2012. Though average tuition and fees during this period increased from \$3,492 in 2006 to \$6,317 in 2013 in nominal terms, UCF still has the lowest tuition of any research university in Florida.

As UCF administrators emphasized to us, because of the gradual and multipronged nature of institutional change, it is difficult to tease out how UCF's various initiatives have contributed to these university-wide changes. Nevertheless, it is possible to compile some data about each initiative that are suggestive of its effects.

In 2013-2014, students earned more than 200,000 scholastic credit hours through Regional Campus programs, a 35 percent increase since 2008-09. Over this period of time, increasing numbers of students have engaged with Regional Campuses through online courses: between 2009 and 2014, Regional Campus online credit hours increased by nearly 77 percent, while Regional Campus face-to-face credit hours decreased by 23 percent. Still, the ability to take face-to-face courses at Regional Campus sites seems to help students graduate faster, especially transfer students. In 2014, 67 percent of transfer students who earned at least half of their face-to-face credits from one of the Regional Campus sites earned their bachelor's degree within two years after transferring, compared to 51 percent of transfer students who took most of their face-toface courses at the main campus.

Both within and outside of Regional Campuses, nearly all of UCF's credit hour growth in the past ten years has come from Online@UCF. In academic year 2002-2003, UCF students completed just under 1 million credit hours, and online credit hours made up 9 percent of this total. In academic year 2014-15, total credit hour production had increased to 1.5 million, and online and blended credits comprised 38 percent of this 
total. In 2014-2015, nearly 78 percent of all (undergraduate and graduate) students at UCF enrolled in an online or blended course. In the fall of 2014, nearly 8,000 students took no face-to-face courses at all.

Success rates are remarkably high and withdrawal rates are remarkably low across all classes at UCF, and vary little by modality. Because of the difficulty of comparing learning outcomes across disciplines, RITE researchers define course success as earning grades of A, B, or C in a course. Based on aggregate data for 2008 to 2011, Students in face-to-face courses have an average success rate of 87.7 percent and an average withdrawal rate of 3.1 percent. Students in fully online courses have a higher average success rate of 88.3 percent and a higher average withdrawal rate of 4.3 percent. Students in blended courses earn higher grades and are less likely to withdraw than students in either of the other modalities, with an average success rate of 90.8 percent and an average withdrawal rate of 2.8 percent.

Online@UCF's focus on instructional quality and faculty development has paid off in terms of student satisfaction. According to surveys administered by RITE, roughly 50 percent of students report being satisfied with their course regardless of course format. In fact, RITE's analyses of student evaluations indicate that modality has little impact on whether a student gives a course an overall rating of "excellent." Instead, the main factor behind an "excellent" rating is the quality of faculty instruction: 99 percent of students who report that their instructor does an excellent job of achieving course objectives, creating a learning environment, and communicating ideas give the course an overall rating of excellent.

Through the lens of the iron triangle, one critical feature of Online@UCF is that it has allowed UCF to increase enrollment and expand instruction with limited physical expansion. UCF's Office of Space Planning, Analysis, and Administration estimates that it would have cost \$329.1 million to build the classroom space needed to offer UCF's online and blended courses face-to-face, plus an additional \$15.4 million per year for maintenance. ${ }^{14}$ Furthermore, students in online courses do not need parking spaces, which cost an astonishing $\$ 12,500$ each to construct. Annual revenue generated by distributed learning course registrations, including tuition, state support, and an \$18 Distance Learning Course fee totaled \$145.66 million in fiscal year 2013-2014, representing close to a 19:1 return on investment, excluding faculty salaries. ${ }^{15}$

\footnotetext{
${ }^{14}$ Blended courses at UCF meet in classroom space roughly one-third as often as fully live courses.

15 “Access, Quality, Efficiency through Online Learning Academic Year 2013-2014," University of Central Florida.
} 
There is also evidence that Online@UCF has transformed UCF's culture. Most UCF students take courses in multiple modalities each term; as the RITE survey research suggests, students appear to be agnostic to modality. Nearly two-thirds of faculty make substantive use of online modalities, ranging from designing and teaching fully online courses to using interactive tools in the Canvas learning management system. As discussed above, academic departments work with CDL to offer an ever-expanding catalog of online and blended courses, and many give credit for research on online teaching in their tenure and promotion considerations. Based on UCF's own research and our observations, Online@UCF is extraordinarily well-integrated into the academic fabric of UCF.

DirectConnect to UCF has also contributed to UCF's enrollment increase while improving graduation rates and reducing time to degree for students who transfer from UCF's partner colleges. To date, 36,942 students have come to UCF through DirectConnect to UCF. Each year, DirectConnect to UCF students make up roughly 66 percent of transfer students (who make up more than 50 percent of UCF's student body). Sixty-seven percent of transfer students who entered UCF in the fall of 2009 graduated by 2014 . This graduation rate is nearly equal to the 68 percent six-year graduation rate of FTIC students. ${ }^{16}$

\section{There is evidence that DirectConnect to UCF has also changed the value proposition for students entering the partner state colleges.}

There is evidence that DirectConnect to UCF has also changed the value proposition for students entering the partner state colleges. For example, some students at Valencia College (the largest partner) report that DirectConnect to UCF was their reason for enrolling at Valencia. ${ }^{17}$ For these students, a bachelor's degree from UCF was the goal, but grades, financial circumstances, or geographical constraints meant they could not enroll there as first-time freshmen. Enrolling at Valencia and "DirectConnect-ing" provided them with an affordable and accessible pathway to the UCF degree. Recognizing the value of the program, the state college partners advertise it extensively:

\footnotetext{
${ }^{16}$ Information provided by UCF's Director of Institutional Research.

17 "DirectConnect to UCF Student Focus Groups: A Qualitative Study," (November 2012), http://www.collegeaccess.ucf.edu/download/attachments/360479/DirectConnect\%20to\%20UCF\%20Student\%20Focus\%2 0Groups.pdf?version=1\&modificationDate=1367511666830\&api=v2.
} 
we saw several billboards cross-promoting DirectConnect to UCF and Valencia College in the Orlando area.

\section{Success Factors}

UCF's two broad strategies for enhancing access, quality, and affordability_building internal capacity and leveraging external capacity - display a number of common characteristics that help explain their success.

\section{Instructional Focus}

UCF is both very large and heavily reliant on online modalities, two features that would lead many observers to question the quality of instruction. However, UCF has placed instruction at the center of its efforts to increase scale. Using technology, while a crucial part of this effort, has served as a means through which to accomplish instructional goals, rather than an end-goal in and of itself. For example, the training required of instructors who incorporate online modalities makes course design-rather than online learning - its primary focus. Similarly, curriculum alignment and instructional improvement have been the primary collaborative activities of UCF and its DirectConnect to UCF partners. The seamless transfer pathway became a catalyst for ensuring that the state colleges were preparing their students to succeed academically at UCF, and that UCF is prepared to help them succeed when they arrive.

UCF's instructional focus has helped to bolster the quality dimension of the iron triangle. It has also made faculty real partners in the effort. We heard from numerous interviewees about the mutual trust between administrators and faculty at UCF, and about the enthusiasm with which faculty seek out CDL resources. We also observed firsthand the deep engagement of UCF faculty with their colleagues from state colleges in the DirectConnect to UCF curricular alignment process at the Access Summit.

\section{Leading through Support}

A key feature of UCF's initiatives is that the administration's primary role is to facilitate and support faculty in the work. One of our interviewees aptly referred to this as "leading through support": rather than having a separate administrative team design curriculum or academic policy for faculty to implement, UCF has provided structures and resources to support faculty members in doing the design work themselves. To succeed with this approach, UCF has had to make participation in the support programs and design work compelling for faculty. It has done this by including deans and department chairs in planning and recruitment, by incorporating expectations of participation into promotion 
and tenure standards, by requiring training as a condition of designing Online@UCF courses, and by linking participation with opportunities to conduct publishable instructional research.

\section{Embracing Partnership}

In his inaugural address in 1992, President Hitt sought to redefine UCF as the "partnership university." At the core of this approach is the belief that UCF is in service to the region, and that it can better serve the region by working in close collaboration with businesses, government, and other educational and cultural institutions than it can on its own. This emphasis on partnership has been behind efforts like the construction of joint use facilities shared by state colleges and Regional Campuses, an ambitious new project with Valencia College and the City of Orlando to redevelop a swath of downtown as an education and technology hub, and of course DirectConnect to UCF.

Our visit to the DirectConnect to UCF College Access Summit revealed that key to UCF's success is a collaborative approach to sustaining these partnerships. As discussed above, DirectConnect to UCF is more than a transfer agreement. It is a set of shared responsibilities amongst staff and faculty at multiple institutions. These responsibilities are supported and reinforced through formal processes-like the College Access Summit-that ensure broad-based communication across institutions. Many of UCF's partnerships, from its Regional Campuses to its industry partnerships, have been grounded in this collaborative and regionally-focused approach.

\section{Long-term view}

While President Hitt and his team certainly had an ambitious vision, and took advantage of opportunities along the way, they have mostly allowed UCF's initiatives to develop organically over time. For example, soon after their implementation, it became clear that online courses would be a means for UCF to realize its ambitions to scale. Yet UCF administrators took a guiding —rather than intervening —role in Online@UCF's growth. They allowed it to develop over decades as a time-intensive, instructionally oriented process, rather than taking short cuts to speed the process of scaling. Over time, Online@UCF has achieved massive scale, but, because of this approach, it rests on a solid instructional foundation.

Consistency in leadership at UCF has been crucial to maintaining this long-term perspective. President Hitt has led UCF for nearly 25 years. J oel Hartman, the Vice Provost whose team manages Online@UCF, has been in his position for nearly as long. The longevity of these and other key leaders has provided the stability for the initiatives to develop in a more deliberate and gradual way. 


\section{Remaining Challenges}

UCF continues to tackle ambitious new goals, further testing the limits of the iron triangle. There are plans to continue to increase enrollment, mainly through providing a larger share of credits through Online@UCF. While UCF has maintained its requirement for faculty to receive intensive training in the development of online courses, its continued growth may put pressure on that commitment. UCF's experimentation with adaptive learning software has the potential to be another means to ensure high-quality, personalized instruction at scale. But it is also a new technology that demands significant investment of time and money, with an uncertain payoff.

The popularity of Online@UCF has raised questions about the role of the Regional Campuses. Since UCF launched Online@UCF in the mid 1990s, students at UCF's Regional Campuses have taken in-person, blended, and online courses to complete their degrees, depending on what works best for their schedules and circumstances. In recent years, increasing shares of students have taken more online courses and fewer face-toface courses; currently online credit hours represent 80 percent of total credit hours completed at Regional Campuses. In response to these changes, UCF's Regional Campuses have shifted their focus to emphasize support services rather than classroom instruction, particularly in connection with DirectConnect to UCF. To reflect this changing reality, Regional Campuses is currently working with Online@UCF to create an online DirectConnect to UCF program that would allow students to transfer to UCF without ever leaving their homes. In this program, Regional Campuses would act, as they often already do, as centers where online students could seek advising and support services without having to travel to UCF's main campus.

While UCF transfer students graduate at nearly the same rate as FTIC students, there is some variation across departments. Faculty and administrators in the College of Engineering and Computer Science, for example, have worked closely with peers at the state colleges to develop a virtually seamless curriculum across institutions. Because of the requirements and pre-requisites of engineering majors, there is little room for error. There is also selection-engineering students tend to plan for a major in the discipline before they even enroll at a partner college. By contrast, students often select a business major later in their academic careers. Perhaps because of this, a larger share of transfer students enter the College of Business Administration with unfulfilled prerequisites.

Faculty and staff work on these kinds of issues at the College Access Summit and other collaborative convenings, but communication in between these sessions is not as effective as it could be in every discipline. One initiative to smooth the transition process is a data-sharing agreement between UCF and Valencia College, its largest DirectConnect partner. While likely to help UCF advisors target services for potential 
transfer students, this initiative also represents a significant undertaking that will require considerable logistical and technical coordination.

Finally, the policy environment in Florida continues to evolve in ways that complicate UCF's work. For example, a recent Florida state policy made developmental courses optional for all recent high school graduates, which is likely to affect the number and the preparation of associate's degree graduates. ${ }^{18}$ With a majority of UCF's students transferring from state colleges, this change will require a great deal of instructional and logistical adaptation by UCF and its DirectConnect to UCF partners.

\section{Conclusion}

UCF has arguably come closer than any institution in the country to breaking the iron triangle of cost, quality, and access. It is the second-largest university in the country, and the least expensive and yet one of the most effective in Florida. It has grown and kept costs low by expanding its instructional capacity through online learning and opening its doors wide to state college transfers. At the same time, it has sought to enhance quality by grounding both of those initiatives in processes of instructional improvement.

Though these changes have developed over the course of twenty years at UCF, they have been guided by intentionally designed processes. In addition to emphasizing instructional quality, these processes embrace regional partnerships and provide real resources and attention to training and support. UCF has directly engaged and earned the support of faculty, staff, and regional partners, while building a culture that celebrates access and innovation. This gradual yet intentional approach to institutional change has laid a solid foundation for UCF to continue to offer high-quality, affordable higher education to more and more students.

${ }^{18}$ See Ashley A. Smith, "When You're Not Ready," Inside Higher Ed (June 25, 2015), https://www.insidehighered.com/news/2015/06/25/floridas-remedial-law-leads-decreasing-pass-rates-math-and-english. 


\section{Appendix}

We conducted interviews with the following UCF administrators, staff, and faculty on April 23 and 24, 2015:

» Tom Cavanagh, Associate Vice President of Distributed Learning

» Pam Cavanaugh, Associate Vice Provost, Student and Academic Initiatives

» Chuck Dziuban, Director of the Research Initiative for Teaching Effectiveness

» Linda Futch, Department Head, Course Design and Delivery

» J oel Hartman, Vice Provost for Information Technologies and Resources

» J eff J ones, Vice Provost for Regional Campuses

» Aaron Misiano, Supervisor, Information Technology, Regional Campuses

» Patsy Moskal, Associate Director of the Research Initiative for Teaching Effectiveness

» Angela Peterson, Associate Vice President, Student Services and Advising, UCF Regional Campuses

» J ennifer Sumner, Director of Academic Services \& Bachelors of Applied Sciences, Regional Campuses

» Kelvin Thompson, Associate Director, Center for Distributed Learning

» Dale Whittaker, Provost

» UCF and partner college faculty, staff and administrators in attendance at the College Access Summit 